(6) OPEN ACCESS

\title{
Image-based teleconsultation using smartphones or tablets: qualitative assessment of medical experts
}

\author{
Constance Boissin, ${ }^{1}$ Lisa Blom, ${ }^{1}$ Lee Wallis, ${ }^{2}$ Lucie Laflamme ${ }^{1,3}$
}

\begin{abstract}
- Additional material is published online only. To view please visit the journal online (http://dx.doi.org/10.1136/ emermed-2015-205258).

${ }^{1}$ Department of Public Health Sciences, Karolinska Institutet, Stockholm, Sweden ${ }^{2}$ Division of Emergency Medicine, Faculty of Medicine and Health Sciences, Stellenbosch University, Bellville, South Africa ${ }^{3}$ University of South Africa, Pretoria, South Africa
\end{abstract}

\section{Correspondence to} Constance Boissin, Department of Public Health Sciences, Karolinska Institutet, Widerströmska Huset, Tomtebodavägen $18 \mathrm{~A}$, Stockholm 171 77, Sweden; constance.boissin@ki.se

Received 24 July 2015 Revised 15 June 2016 Accepted 17 August 2016 Published Online First 5 October 2016

\section{(1) crossank}

To cite: Boissin C, Blom L, Wallis L, et al. Emerg Med J 2017:34:95-99.

\begin{abstract}
Background Mobile health has promising potential in improving healthcare delivery by facilitating access to expert advice. Enabling experts to review images on their smartphone or tablet may save valuable time. This study aims at assessing whether images viewed by medical specialists on handheld devices such as smartphones and tablets are perceived to be of comparable quality as when viewed on a computer screen.
\end{abstract}

Methods This was a prospective study comparing the perceived quality of 18 images on three different display devices (smartphone, tablet and computer) by 27 participants (4 burn surgeons and 23 emergency medicine specialists). The images, presented in random order, covered clinical (dermatological conditions, burns, ECGs and X-rays) and non-clinical subjects and their perceived quality was assessed using a 7-point Likert scale. Differences in devices' quality ratings were analysed using linear regression models for clustered data adjusting for image type and participants' characteristics (age, gender and medical specialty).

Results Overall, the images were rated good or very good in most instances and more so for the smartphone $(83.1 \%$, mean score 5.7$)$ and tablet $(78.2 \%$, mean 5.5$)$ than for a standard computer $(70.6 \%$, mean 5.2$)$. Both handheld devices had significantly higher ratings than the computer screen, even after controlling for image type and participants' characteristics. Nearly all experts expressed that they would be comfortable using smartphones $(n=25)$ or tablets $(n=26)$ for image-based teleconsultation.

Conclusion This study suggests that handheld devices could be a substitute for computer screens for teleconsultation by physicians working in emergency settings.

\section{INTRODUCTION}

Mobile health (mHealth) is increasingly acknowledged as a means to improve healthcare delivery globally. ${ }^{1}$ By speeding up and facilitating access to expert advice, it contributes to effective treatment, reduced referral rates and ultimately reduced costs for both healthcare systems and patients. ${ }^{2}{ }^{3}$ In addition, increasing smartphone utilisation and rapidly growing internet access worldwide make mHealth more widely available, including in resource-poor settings. Thus, mHealth may have a promising impact on the reduction of inequalities in access to healthcare.

Image-based mHealth in particular is an area that is developing rapidly, allowing clinicians at point of care to take and transmit pictures to seek expert advice. ${ }^{145}$ The practice is valuable for pictures taken of existing ECGs or radiological images ${ }^{6} 7$

\section{Key messages}

What is already known on this subject?

- Studies from radiology and echocardiography show that experts can accurately make a diagnosis using images viewed on handheld devices.

- Experts viewing radiological images on tablets perceive them as of comparable quality as when viewed on computer screens.

- Whether those favourable perceptions are specific to tablets as a handheld device or to the particularity of those clinical fields remains to be determined.

What this study adds

- Images viewed on handheld devices, on both smartphones and tablets, are perceived by medical experts as of better quality than when viewed on a computer screen.

- Smartphones and tablets outperformed standard, non-radiological computers for ECGs, $X$-rays, clinical photographs of dermatological conditions and burn wounds.

and in conditions like ophthalmology, ${ }^{8}$ dermatol$\mathrm{ogy}^{9}$ and burns, ${ }^{10}$ where pictures can be taken of a specific body region of concern. However, to ensure effectiveness, the turnaround time has to be kept to a minimum. This may require experts to view the images received on their handheld smartphone or tablet to avoid potential delays incurred should access to a computer screen be required. Promisingly, in radiology and echocardiography where practice is in essence based on image analysis, recent studies demonstrate that experts can accurately diagnose images on tablets. ${ }^{11}{ }^{12}$ Further, an additional study from the radiological field reveals that experts' subjective evaluation of the quality of radiological images is as positive for tablets as for computer screens. ${ }^{13}$

The evidence is promising regarding the usability of handheld devices (smartphones and tablets) for expert teleconsultation. But it remains to be determined whether these observations are specific to radiological images or to the familiarity of the radiologists-or any expertise relevant to emergency medicine-with the type of images presented.

This study is concerned with acute conditions prevalent in resource-poor settings, specifically burns, and assesses whether images viewed on handheld devices are perceived by potential experts as being comparable in quality as when viewed on a standard, non-radiological computer screen. 


\section{METHODS}

\section{Intervention}

In this prospective study, 18 images were viewed in a random order on three different display devices by 27 participants.

Three different display devices of types commonly used by physicians to view images were selected: a laptop computer screen (model Lenovo IdeaPad G5070 i3 $1.9 \mathrm{GHz}$, Windows 8 with a $15.6^{\prime \prime}$ HD LED screen of resolution $1366 \times 768$ pixels and a pixel density of $100 \mathrm{pixel} / \mathrm{inch}$ (PPI)) used as the reference, a tablet (model Samsung Galaxy Tab 3 with a $10.1^{\prime \prime}$ screen of resolution $1280 \times 800$ pixels and a pixel density of 149.45 PPI) and a smartphone (model Apple iPhone 5S with a $4^{\prime \prime}$ screen of resolution $1136 \times 640$ pixels and a pixel density of 326 PPI).

We used a selection of 18 images covering both clinical and non-clinical subjects (figure 1). The images were obtained from three different sources: two previous studies from our research group ( $\mathrm{n}=4$ and 6 , respectively) ${ }^{10} 14$ and from an open access online medical database $(n=8) .{ }^{15}$ The clinical images included dermatological conditions and burns captured with a camera as well as images of ECGs and standard plain film X-rays that represent results of examinations and are therefore not a direct photograph of a body part.

\section{Participants}

A purposive sampling was used to recruit 27 participants based on two inclusion criteria. First, participants reported being likely to be contacted in their professional life to give expert opinion on acute burn injuries. Second, they reported having normal visual acuity and colour vision. They were enlisted during two consecutive expert meetings that took place in April 2015 in Cape Town, South Africa; during a local meeting focusing on burn injuries and during an international emergency medicine conference. A total of four South African burn surgeons and 23 emergency medicine specialists practicing in Sub-Saharan Africa or in the USA were enrolled. All but one declared having at least moderate experience in acute burn care during the survey.

\section{Survey}

A questionnaire was developed using the online software SurveyMonkey. Each individual participant viewed the 18 images on each of the three devices and was asked to rate the overall quality of each image on a 7 -point Likert scale $(1=$ terrible to 7 =excellent). Beforehand, the participants were instructed to focus on the quality of the images as such rather than on the ability to diagnose any particular condition. The images were presented in a random order as defined automatically by the software. Once participants had rated all the images on each device, two questions were asked concerning image quality and three regarding how frequently the participant used the specific type of device to look at images for personal, professional and teleconsultation purposes, the use of the device's zoom feature during the survey and whether they would feel comfortable using the device for image-based remote

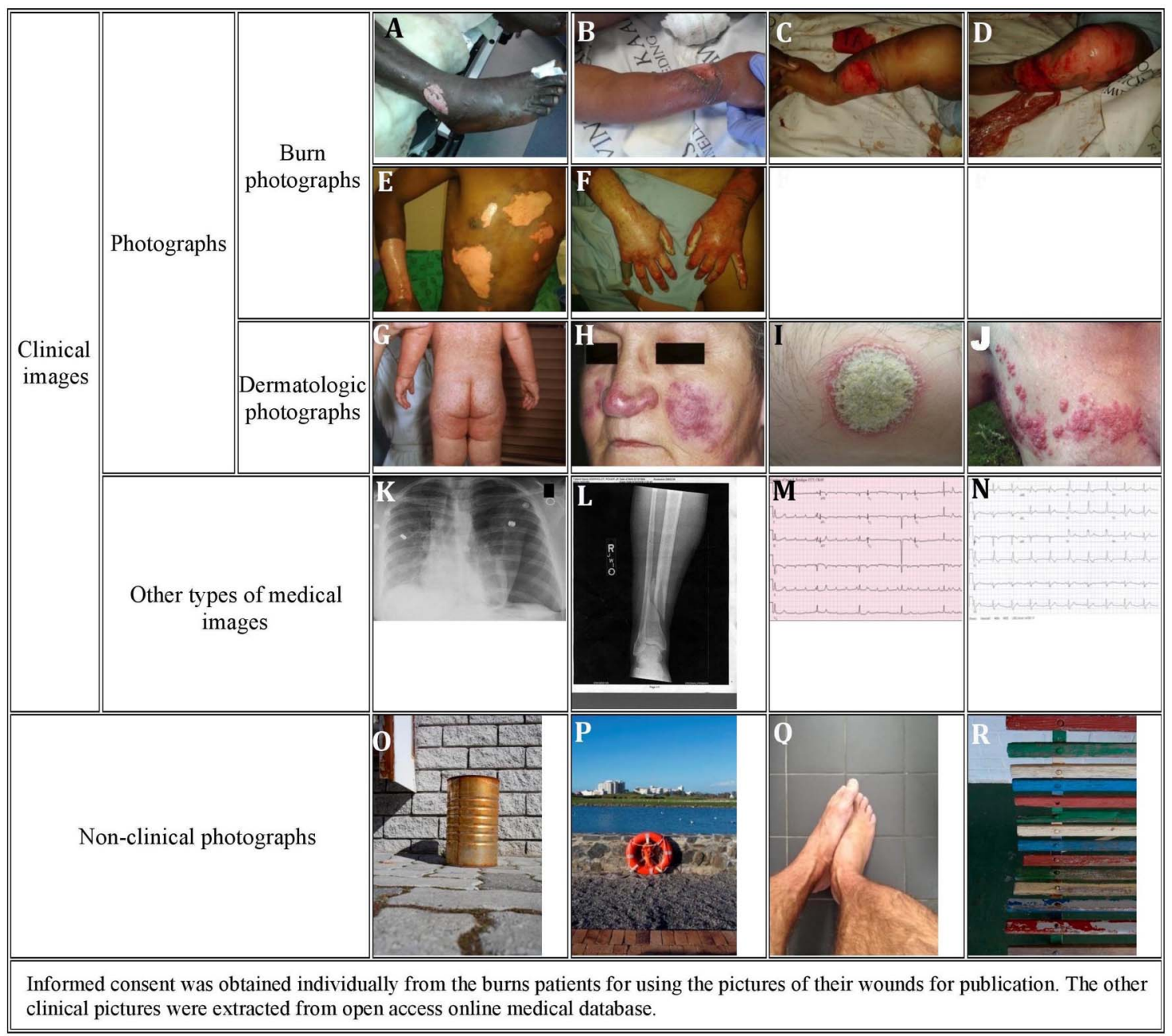

Figure 1 The 18 images presented in the survey. 
consultation. Concerning quality, the participants were asked to rank order the importance of five image features: ${ }^{14}$ focus, resolution, contrast, colour and composition and then how they interpreted the word 'quality' when completing the survey. Demographic data were collected at the very end.

\section{Data collection procedure}

Each participant was tested individually and seated at a defined position where ambient lightning would be consistent and was presented with each device one at a time. Although it was practically not feasible to hide what type of device they were presented with, each device was placed in a custom-designed cover to hide the brand or model. All three were set to maximum luminance levels. The order in which the participants were assigned to the devices was predetermined in accordance with the six possible permutations of the devices.

\section{Data analysis}

A linear regression procedure for data clustered by participant was applied, where quality rating scores for the tablet and the smartphone were compared with those for the computer screen. We then used a Wald-type test to assess the interaction between the type of device and, in turn, the type of image and medical specialty of the participants by adding their product terms in the model and jointly testing the regression coefficients of their product terms equal to zero. The interaction between type of device and medical specialty was not significant $(\mathrm{F}=1.46$; $\mathrm{p}=0.25$ ) and medical specialty was not considered further. By contrast, the interaction between type of device and type of image was significant $(\mathrm{F}=6.47 ; \mathrm{p}<0.001)$ and we therefore performed similar regression analyses stratified by image type: burns, dermatology, other clinical images (ECGs and X-rays) and non-clinical images. All data treatments were performed using Stata V.12.

\section{Ethical considerations}

The study was approved by the Human Ethics Research Committee at the Stellenbosch University (\#N15/03/018). The participants' consent was obtained electronically prior to fulfilling the first survey.

\section{RESULTS}

Table 1 presents the demographic characteristics of the participants, with a mean age of 38 years and $41 \%$ being women. The device that was used most often by the participants for personal and professional purposes was the smartphone. All but one used a smartphone at least a few times a week for personal purposes and 23 participants used a smartphone for professional purposes. The computer was used by 25 and 22 participants for personal and professional purposes, respectively, and the tablet was used by 19 and 15 participants for personal and professional purposes, respectively. A total of 19 participants reported using their smartphones specifically for image-based teleconsultation at least a few times a month compared with 9 participants for tablets and 11 participants for computers.

Table 2 presents the results of the linear regression models comparing quality ratings for the tablet and the smartphone with the computer for all images aggregated and by type of image. Overall, both the tablet and the smartphone have significantly higher ratings than the computer. The tablet and smartphone have higher ratings than the computer for all specific types of image, with the exception of burns where there was no significant difference between the tablet and computer and of
Table 1 Demographic characteristics (gender and age) of the participants by medical specialty

\begin{tabular}{lcc}
\hline Variable & $\begin{array}{l}\text { Burn surgeons } \\
(\mathbf{n}=4)\end{array}$ & $\begin{array}{l}\text { Emergency medicine specialists } \\
(\mathbf{n}=23)\end{array}$ \\
\hline $\begin{array}{l}\text { Gender } \\
\text { Male, } \mathrm{n}(\%)\end{array}$ & $2(50 \%)$ & $14(61 \%)$ \\
$\quad$ Female, $\mathrm{n}(\%)$ & $2(50 \%)$ & $9(39 \%)$ \\
$\begin{array}{l}\text { Age } \\
\quad \text { Median (min-max) }\end{array}$ & $42.5(36-73)$ & $36(30-45)$ \\
\hline
\end{tabular}

Table 2 Mean difference $(95 \% \mathrm{Cl})$ in quality rating scores between the tablet and the smartphone compared with the computer using linear regression models for clustered data for all images aggregated and by image type

\begin{tabular}{|c|c|c|c|}
\hline Image category & Computer & $\begin{array}{l}\text { Tablet } \\
\text { Mean difference } \\
(95 \% \mathrm{Cl})\end{array}$ & $\begin{array}{l}\text { Smartphone } \\
\text { Mean difference } \\
(95 \% \mathrm{Cl})\end{array}$ \\
\hline $\begin{array}{l}\text { All images } \\
(n=1458)\end{array}$ & 0 (ref) & $0.38(0.20$ to 0.55$)$ & $0.49(0.29$ to 0.70$)$ \\
\hline Burns $(n=486)$ & 0 (ref) & $0.08(-0.15$ to 0.31$)$ & $0.54(0.30$ to 0.77$)$ \\
\hline $\begin{array}{l}\text { Dermatology } \\
(n=324)\end{array}$ & 0 (ref) & $0.77(0.38$ to 1.16$)$ & $0.65(0.28$ to 1.01$)$ \\
\hline $\begin{array}{l}\text { Other clinical } \\
\text { images }(n=324)\end{array}$ & 0 (ref) & $0.39(0.14$ to 0.63$)$ & $0.31(-0.03$ to 0.66$)$ \\
\hline $\begin{array}{l}\text { Non-clinical } \\
\text { images }(n=324)\end{array}$ & 0 (ref) & $0.42(0.16$ to 0.67$)$ & $0.45(0.20$ to 0.71$)$ \\
\hline
\end{tabular}

other clinical images, where no difference was found between the smartphone and the computer.

Figure 2 presents a box-and-whisker plot of the participants' quality ratings for each image stratified by device. Altogether, the images were rated good or very good in many instances and more so for the smartphone (83.1\%, average score of 5.7) and the tablet $(78.2 \%$, average of 5.5$)$ than for the computer $(70.6 \%$, average of 5.2$)$. There were wide variations in ratings for each image and within each type of image. When looking at the ratings between image types, the differences in quality ratings were larger within the photograph-based clinical fields (namely between burns and dermatology) than between the photographs and image-based ones or even between all clinical and non-clinical images.

It is of note that participants used the zoom function more often with the smartphone $(n=22)$ than with the tablet $(n=10)$ and the computer $(\mathrm{n}=8)$. In addition, among the features suggested as determinant of their judgement on image quality, resolution and focus were ranked as either most or second most important by $67.1 \%$ and $55.7 \%$ of the participants respectively, which contrasts with $67.5 \%$ ranking composition as least important (figure 3). Also, when asked to define the quality of a picture, one in four participants $(n=7)$ answered that quality was when a picture allowed them to make a clear diagnosis; this is in spite of having viewed images that were not clinical at all. Furthermore, in their definitions, most did not specify any technical features but others $(\mathrm{n}=8)$ underlined resolution or clarity. Almost all participants answered that they would be comfortable or very comfortable giving image-based clinical advice using the smartphone, tablet and computer (25, 26 and 22 respectively). 


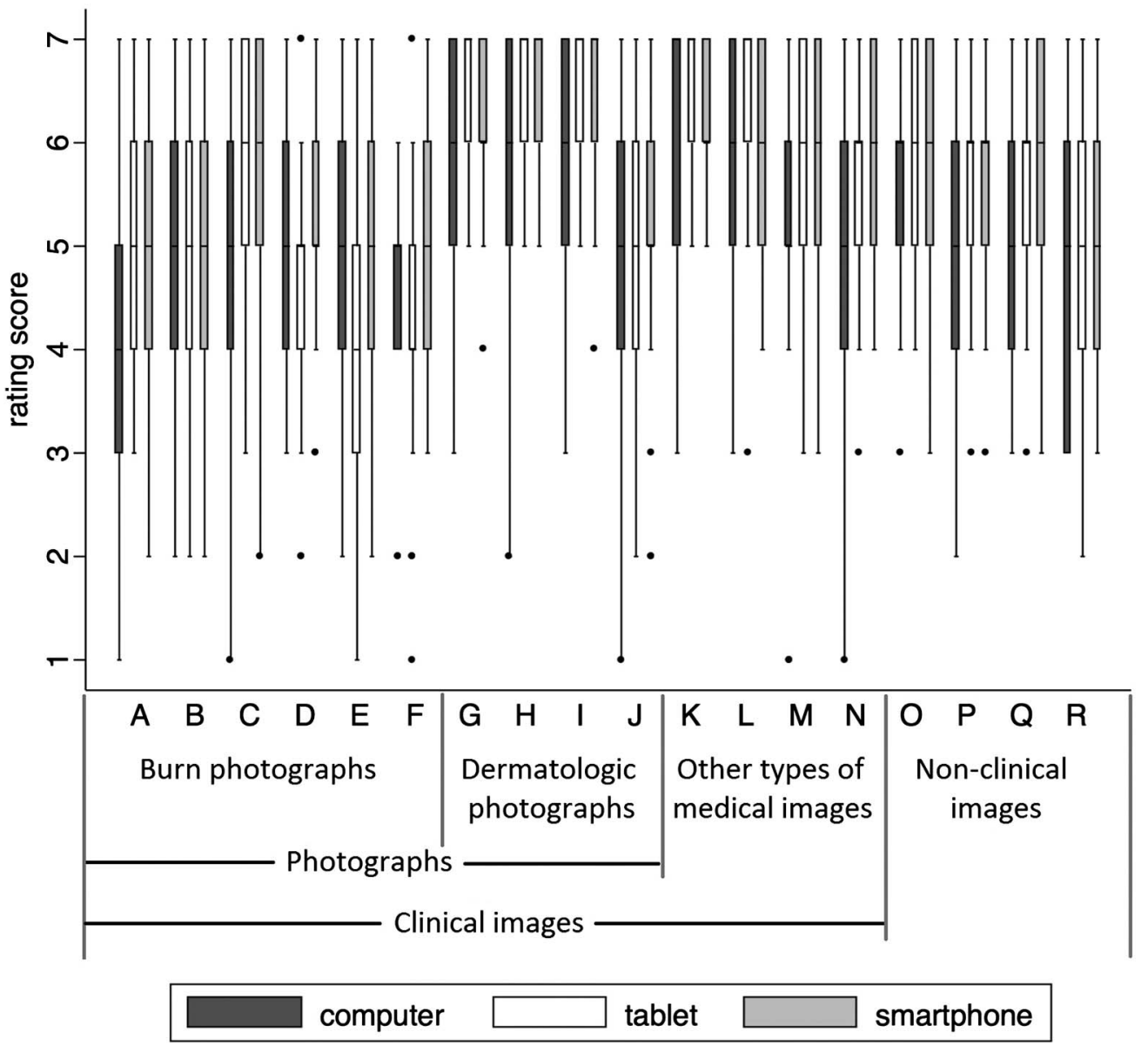

Figure 2 Box-and-whisker plot showing the participants' quality rating scores by image for all three devices.

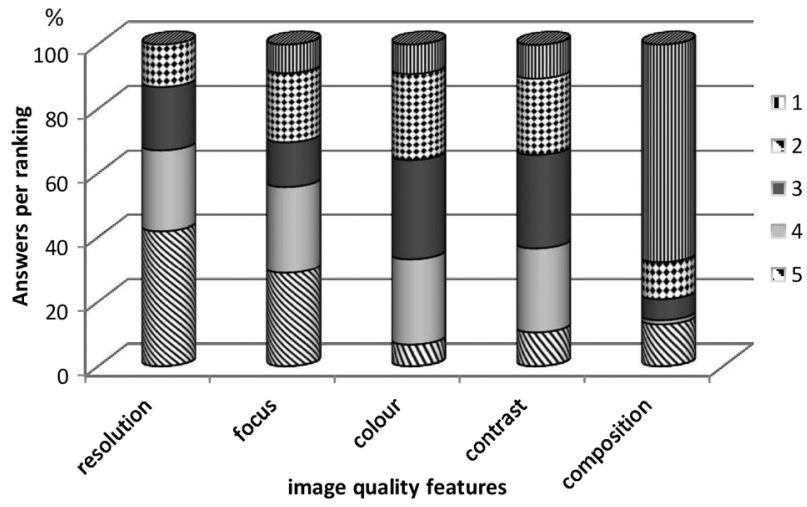

Figure 3 Representation showing the percentage of times image quality features (resolution, focus, colour, contrast and composition) ranked from 1 (least important) to 5 (most important) for all participants and all devices aggregated.

\section{DISCUSSION}

\section{Main findings}

This study broadens the current knowledge on the potential for usage of handheld devices in the emergency medicine field where image quality is a key support for diagnostic accuracy. ${ }^{16}$ In the opinion of experts involved in emergency medicine, handheld devices offer a support as good as, if not better than, standard, non-radiological computer screens for viewing images. In fact, almost all experts felt that they would be comfortable using both tablets and smartphones for image-based teleconsultation, regardless of image type: non-clinical and clinical images and, in the latter case, not only ECGs and X-rays but also photographs of burns and other dermatological conditions. It is also noted that the medical specialty of the participants did not substantially influence their ratings.

An earlier study addressed the use of tablets in the field of radiology $^{13}$ and its findings point in the same direction: the tablet was rated equal to or better than the computer screen. To the best of our knowledge no previous study has included a smartphone as a handheld device although it was the most frequent one used by the specialists taking part in this study $(70 \%$ used a smartphone at least once a month for teleconsultation) and may also be among medical professionals in general. ${ }^{17}$

The computer screen's spatial resolution was lower than that of the handheld devices and this may be one of the reasons why it received a lower quality rating. However, spatial resolution is just one of the technical features that come into play when looking at display quality ${ }^{18}$ and its importance may depend on the type of image. ${ }^{19}$

The large variations found within each image category and more so between the clinical photographs of the type not studied thus far (burns and dermatological) might enhance the importance of protocols for image capture in those specialties. ${ }^{20}$

We believe that the results are robust as the design adopted included permutation of the devices, randomisation of the images and hidden device brands. The experiment was also conducted in realistic lightning conditions so as to represent an environment as similar as possible to what experts experience in real-life consultation. Further, as the display characteristics 
(spatial resolution, noise and reflectance) of handheld devices have already been assessed technically, ${ }^{18}$ the subjective user approach we adopted felt most relevant.

\section{Limitations}

One potential source of bias in the study is that although the brand of the devices was hidden, it was obvious to the participants what each one of the devices was-smartphone, tablet or computer-and some preconceived opinions in favour of either one of them may have come into play. Controlling for diagnostic accuracy could possibly have provided some further information on this potential bias, although the study was not designed for this purpose. Our sample of experts may also have some weakness as it was purposive, rather than random (which was not possible as we had few burns specialists), and as there was some imbalance between the number of participants from the two specialties represented. Further, although we enlarged the spectrum of specialties relevant to emergency medicine, we did not cover them all.

The resolution of the images tested could not be controlled as the images were obtained from different sources. It is possible that this could have influenced the participants' perceptions of the quality; however, this could also be true in the clinical setting. Regarding the performance of the devices chosen, the laptop model chosen was as recent as possible to ensure optimum screen quality, but we purposely avoided using the most recent models of handheld devices so as to better represent the ones most likely to be routinely used on a daily basis in lowincome and middle-income country settings. Although the results shown in this study are only representative of the devices tested, given the rapid qualitative developments achieved in photographing and transferring images, there are good reasons to expect that similar-perhaps even better-results would be found should more recent models of smartphones or tablets be used.

\section{Implications}

In line with recent research showing that images of quality can be taken with a smartphone, ${ }^{14}$ our results support the notion that handheld devices are a good solution for image-based teleconsultation. The ratings addressed in this study are however limited to a small number of images and it might be that handheld devices are more suitable for occasional advice than for a high load of pictures. ${ }^{19}$ Further, complementary studies on diagnostic accuracy using handheld devices in teleconsultation would be beneficial to confirm the results in specific specialties and settings. Other important aspects to take into consideration are related to legal issues around data-sharing, patient consent, confidentiality and security which need to be addressed prior to use of such devices. ${ }^{19} 21$

\section{CONCLUSION}

Images viewed on handheld devices were rated as having better quality compared with viewing on a computer screen. Thus, this study suggests that handheld devices could be a substitute for computers for image-based teleconsultation in emergency settings, with the potential to save valuable time for the clinicians and perhaps even enhance equity in healthcare.
Contributors $\mathrm{LL}, \mathrm{CB}$ and $\mathrm{LW}$ determined the research question. $\mathrm{LL}$ and $\mathrm{CB}$ elaborated the study design. CB, LB and LW took responsibility for the data collection. CB performed the data analysis. All authors took part in the interpretation of the results and made significant contribution to the manuscript.

Funding This study was funded by the Marianne and Marcus Wallenberg Foundation under the grant number MMW 2011.0138.

Competing interests None declared.

Ethics approval Human Ethics Research Committee at the Stellenbosch University. Provenance and peer review Not commissioned; externally peer reviewed.

Open Access This is an Open Access article distributed in accordance with the Creative Commons Attribution Non Commercial (CC BY-NC 4.0) license, which permits others to distribute, remix, adapt, build upon this work non-commercially, and license their derivative works on different terms, provided the original work is properly cited and the use is non-commercial. See: http://creativecommons.org/ licenses/by-nc/4.0/

\section{REFERENCES}

1 World Health Organization. mHealth: new horizons for health through mobile technologies. WHO Press, 2011.

2 Al-Hadithy N, Ghosh S. Smartphones and the plastic surgeon. J Plast Reconstr Aesthetic Surg 2013;66:e155-61.

3 Betjeman TJ, Soghoian SE, Foran MP. mHealth in Sub-Saharan Africa. Int J Telemed App/ 2013;2013:482324.

4 Vital Wave Consulting. mHealth for development: the opportunity of mobile technology for healthcare in the developing world. UN Foundation-Vodafone Foundation Partnership, 2009.

5 Lemaire J. Scaling up mobile health-elements necessary for the successful scale up of mHealth in developing countries. Advanced development for Africa, 2011.

6 Muhlestein JB, Le V, Albert D, et al. Smartphone ECG for evaluation of STEMI: results of the ST LEUIS Pilot Study. J Electrocardiol 2015;48:249-59.

7 Schwartz $A B$, Siddiqui $G$, Barbieri JS, et al. The accuracy of mobile teleradiology in the evaluation of chest X-rays. J Telemed Telecare 2014;20:460-3.

8 Maamari RN, Keenan JD, Fletcher DA, et al. A mobile phone-based retinal camera for portable wide field imaging. Br J Ophthalmol 2014;98:438-41.

9 Karlsson MA, Lindelöf $B$, Wahlgren CF, et al. Mobile teledermatology is a valid method to estimate prevalence of melanocytic naevi in children. Acta Derm Venereol 2015;95:303-6.

10 Boissin C, Laflamme L, Wallis L, et al. Photograph-based diagnosis of burns in patients with dark-skin types: the importance of case and assessor characteristics. Burns 2015:41:1253-60.

11 Caffery LJ, Armfield NR, Smith AC. Radiological interpretation of images displayed on tablet computers: a systematic review. Br J Radiol 2015;88:20150191.

12 Choi BG, Mukherjee M, Dala $P$, et al. Interpretation of remotely downloaded pocket-size cardiac ultrasound images on a web-enabled smartphone: validation against workstation evaluation. J Am Soc Echocardiogr 2011;24:1325-30.

13 Toomey RJ, Rainford LA, Leong DL, et al. Is the iPad suitable for image display at American Board of Radiology examinations? Am J Roentgenol 2014;203:1028-33.

14 Boissin C, Fleming J, Wallis L, et al. Can We Trust the Use of Smartphone Cameras in Clinical Practice? Laypeople Assessment of Their Image Quality. Telemed e-Health 2015;21:887-92

15 Wikimedia Commons. 2015. http://commons.wikimedia.org/wiki/

16 van der Heijden JP, Thijssing L, Witkamp L, et al. Accuracy and reliability of teledermatoscopy with images taken by general practitioners during everyday practice. J Telemed Telecare 2013;19:320-5.

17 O'Reilly MK, Nason GJ, Liddy S, et al. DOCSS: doctors on-call smartphone study. Ir J Med Sci 2014;183:573-7.

18 Yamazaki A, Liu P, Cheng WC, et al. Image quality characteristics of handheld display devices for medical imaging. PLOS ONE 2013:8:e79243.

19 Hirschorn DS, Choudhri AF, Shih G, et al. Use of mobile devices for medical imaging. J Am Coll Radiol 2014;11:1277-85.

20 Ashique KT, Kaliyadan F, Aurangabadkar SJ. Clinical photography in dermatology using smartphones: An overview. Indian Dermatol Online J 2015;6:158-63.

21 Kunde L, McMeniman E, Parker M. Clinical photography in dermatology: ethical and medico-legal considerations in the age of digital and smartphone technology. Australas J Dermatol 2013;54:192-7. 